\title{
«Sì, sono un lottatore»
}

\section{Daniel Lüthi}

Libero giornalista e fotografo, consulente mediatico, Berna

Un caseggiato sulla strada con il Monte San Salvatore visibile solo da dietro. Nessuna sede di rappresentanza quindi e nessuna aria da "re del villaggio». Il dottore attende già nel corridoio davanti allo studio, con un ampio sorriso su un manifesto elettorale. «Si può fare di più» è lo slogan che in aprile ha permesso al medico che è anche un politico di vincere le elezioni ed essere rieletto nel parlamento cantonale. Questa volta però per un altro partito, una tipica storia «alla Denti».

\section{In lotta contro le casse}

"Qui in Ticino la moglie uno la può cambiare», commenta Denti con il suo tipico sorriso di compiacimento, «il partito no. Io l'ho fatto lo stesso e ho vinto.» Aggiunta importante: «Naturalmente sono rimasto fedele alle mie idee.» Del suo vecchio partito, il PPD, non parla volentieri: «Ha perso il suo spirito sociale e ormai discute solo invece di prendere anche decisioni e impegnarsi veramente per qualcosa. Io cercavo aria fresca e utopie.» Denti si è iscritto ai Verdi dove ora si impegna sui suoi temi fondamentali che, ovviamente, riguardano prevalentemente il settore sanitario. In particolare, si oppone sempre più alle casse malati perché «ci rapinano». La sua opinione è che dal 1996 i ticinesi abbiano pagato alle casse premi molto più elevati del necessario. Secondo uno studio della società medica cantonale, le casse malati avrebbero accumulato a spese del Canton Ticino riserve per oltre 400 milioni di franchi. In alcuni cantoni invece i premi non sarebbero
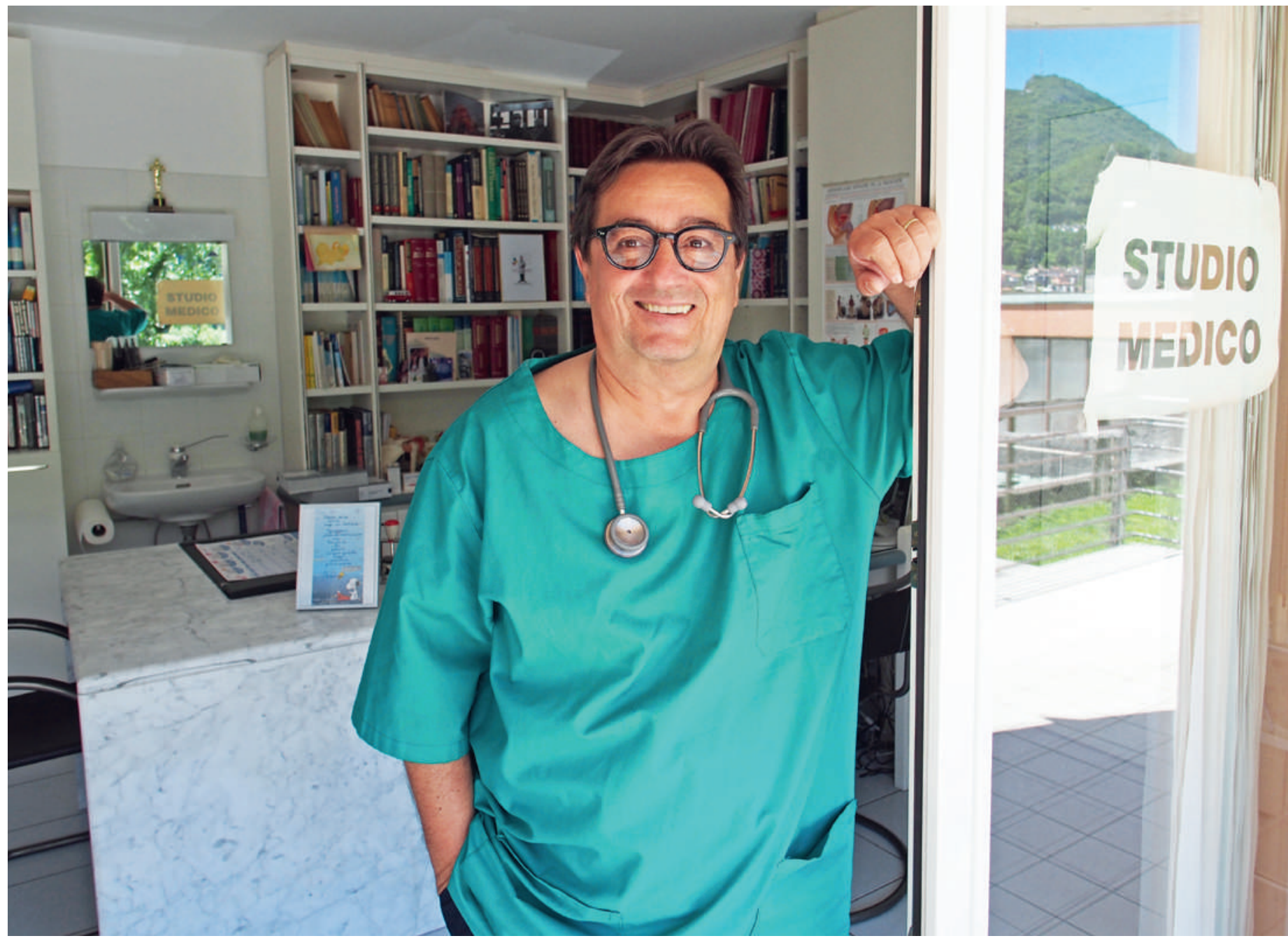
nemmeno sufficienti a coprire le spese correnti. «È urgente separare chiaramente l'assicurazione di base dalle assicurazioni complementari», spiega Denti, che si impegna su diversi punti: valori del punto tariffario più elevati e premi più bassi, quindi a favore dei medici di famiglia e dei pazienti, ma anche del Canton Ticino, che non deve essere una regione periferica.

Risulta così chiaro che il suo nome "Denti» è anche un programma, perché mostrarli gli piace... e non solo quando ride. «Quando sono arrabbiato, sono veramente furioso", spiega con sguardo penetrante "e allora divento molto tenace. Sì, sono un lottatore.» Un aspetto del carattere emerso già in gioventù: «Sono figlio di un operaio, mio padre è morto presto e a chi diceva che uno come me non avrebbe dovuto studiare ho fatto capire che si sbagliava.»

\section{A capo di una corporazione}

Quando sta lì seduto e racconta, appare amabile e rilassato, sicuramente simpatico e non certo aggressivo o rabbioso. "Sono anche un gaudente», racconta, «mi piace moltissimo andare con il mio motoscafo sul mio lago di Lugano oppure fare una passeggiata con il nostro setter irlandese.» Tempo per queste cose però ne ha poco. «Le mie giornate sono lunghe e lavoro volentieri anche di notte.»

Per spiegarlo stampa una poesia di Alda Merini che loda la quiete delle ore notturne. Sonno? «Poco.» Non c'è tempo per tutto. Spiega che lui e sua moglie hanno rinunciato volutamente ad avere figli, perché prendere sul serio le persone significa anche avere tempo da dedicare loro sia nella vita professionale che in quella privata. Per questo può accadere che i pazienti debbano aspettarlo a lungo. Poi però dedica loro il tempo necessario e non gli importa restare in studio anche fino a sera inoltrata.

Oltre al suo studio, Franco Denti ricopre anche numerose cariche. Ad esempio è presidente dell'associazione medica ticinese, che qui non si chiama appunto «associazione» o qualcosa di simile, bensì "ordine» che fa pensare più a una corporazione, una "corporazione orgogliosa», come sottolinea Denti. L'orgoglio ha a che fare anche con lo status sociale: "In una comunità il medico dovrebbe avere un ruolo simile a quello del sindaco, del parroco o dell'insegnante, così almeno era un tempo.» Denti si riferisce al riconoscimento dell'autorità e al rispetto, non all'ammirazione e a un senso di superiorità.

«Io sono vicino alla gente», spiega, «tutti hanno il mio numero di cellulare e il mio numero privato, ma non ne abusano. Inoltre mi portano uova, mele o vino e mi invitano a battesimi e matrimoni.» Nel lungo corridoio

\section{Franco Denti}

II dott. Franco Denti è nato nel 1957 a Sorengo, vicino a Lugano. Ha frequentato le scuole a Lugano e ha studiato medicina a Pavia (Italia), superando I'esame di stato nel 1989. Successivamente ha proseguito la formazione, soprattutto in chirurgia, medicina interna, cardiologia, urologia e gineco-

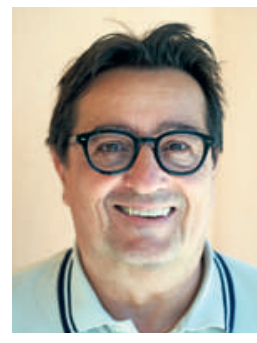

Iogia, presso diversi ospedali nel Canton Ticino e a Varese, Losanna, Berna e Zurigo. Nel 1998 ha svolto I'esame di stato sempre a Zurigo, ottenendo il titolo di medico specialista in medicina generale della FMH. Lo stesso anno ha aperto uno studio medico a Barbengo, nei pressi di Lugano, dove per 60 anni non c'era mai più stato nessun medico. Parallelamente è presidente dell'associazione medica cantonale "Ordine dei Medici del Cantone Ticino", delegato della FMH, membro del comitato direttivo della SSMG e della Fondazione Carnegie, nonché membro del parlamento cantonale ticinese. Franco Denti è sposato e vive con la moglie a Lugano.

dello studio sono appesi in modo del tutto naturale, come in una mostra d'arte, i calendari Pirelli dal 1998 ad oggi, fotografie «cult» di un produttore di pneumatici. Come spiega l'assistente dello studio, le donne mezze nude non le danno fastidio e anzi anche lei apprezza questo periodico regalo di un paziente.

Denti è convinto che, per un medico ticinese, le cose funzionano in parte diversamente rispetto ai colleghi a nord delle Alpi, «il rapporto tra medico e paziente, ad esempio, è più stretto e più personale». In questo contesto Denti usa il termine "cultura medica ticinese»,

\section{«Io sono vicino alla gente.»}

che fa di nuovo balenare una buona dose di campanilismo. Sulla scrivania campeggia infatti una fotografia del FC Malcantone Agno. «Dopo il declino della FC Lugano, volevo contribuire a rafforzare questa squadra e ne ero il medico.» In mezzo c'è l'ex allenatore dei mattatori locali, Vladimir Petković, che oggi allena la nazionale svizzera. «Si può fare di più.»

\section{Un critico, anche tra le proprie file}

Franco Denti si impegna su molti fronti, anche come volontario. Da 25 anni infatti, sempre nel mese di agosto, si reca come volontario a Lourdes con circa 200 pellegrini. Inoltre, è membro del comitato direttivo della Fondazione Carnegie, che ogni anno premia chi salva vite e riceve sostanziosi finanziamenti da facoltosi sostenitori. «È un bene che i ricchi effettuino donazioni», commenta Denti, «ma in realtà non avrebbero dovuto poter diventare così ricchi, avrebbero dovuto distribu- 
ire prima la propria fortuna.» Denti è uno spirito critico, anche tra le proprie file. Ad esempio, ha lottato contro il progetto Managed Care sostenuto invece da molti suoi colleghi. «Il progetto era troppo favorevole alle casse malati. Anche io voglio le reti, ma secondo criteri che siano utili al corpo medico e ai pazienti, non alle casse.»

Denti critica perfino le proprie reti, come la SSMG che in agosto si vuole fondere con la SSMI oppure l'associazione «Medici di famiglia Svizzera». «Lì il Ticino non è rappresentato, non ci vogliono. Inoltre, troppo spesso sono disposti a compromessi a un livello troppo basso. Così non si ottiene nulla.»

\section{«Si può fare di più.»}

A volte però il lottatore Denti fa trapelare anche un po' di delusione e disillusione, proprio come i politici del partito che ha appena lasciato. A livello politico si è impegnato a favore di condizioni quadro migliori per $\mathrm{i}$ medici di famiglia, spiega, e alcuni risultati sono stati raggiunti. "Ora però, che abbiamo strutture migliori, ci mancano sempre più medici che vogliono dedicarsi alla medicina di famiglia.» Sono i punti ben noti, di cui anche Denti si sta occupando: «I giovani medici vogliono lavorare di meno, con più regole e guadagnando di più. A questo proposito abbiamo un problema di motivazione.»

Per questo, secondo Denti, proprio nelle regioni periferiche è difficile trovare successori per gli studi medici. Come racconta, quando allora è arrivato piuttosto casualmente a Barbengo ha aperto uno studio come medico di base in un paesino dove per 60 anni non c'era più stato nessun medico. «Nel giro di due anni ho raggiunto gli obiettivi che nel mio business plan avevo previsto dopo cinque anni.» Oggi il suo archivio com- si reca da lui e in estate ha come pazienti anche molti turisti, soprattutto della Svizzera tedesca e tedeschi. Le visite a domicilio fanno parte del lavoro quotidiano.

\section{Vecchi obiettivi, nuove piattaforme}

Il futuro tuttavia è incerto. Attualmente nel suo studio lavora anche un infettivologo italiano. Per lui si tratta di un'opportunità di specializzazione e per conoscere la situazione svizzera, mentre per Denti, con tutti i suoi impegni, è un prezioso aiuto. Chissà che un giorno, magari, il collega dell'Italia settentrionale rilevi lo studio? Franco Denti alza le spalle e si dice moderatamente fiducioso.

Comunque sia, Denti non sarebbe Denti se non individuasse sempre nuove piattaforme per le proprie iniziative. «Io sono un pragmatico e mi piace lavorare a dei progetti, il contesto può anche cambiare», spiega. Così, dopo il fallimento della cassa unica, sta lavorando tra l'altro al progetto di continuare a gestire una cassa intercantonale. Con riferimento ai suoi mandati politici il suo prossimo obiettivo è a Berna, a livello nazionale. Alle prossime elezioni federali in autunno, il medico e politico dei Verdi del Ticino, sarà candidato al Consiglio degli Stati o al Nazionale, restando fedele al suo motto «Si può fare di più». Oppure, come dice per finire: «Chi vuole fare e mettere in moto qualcosa trova sempre un modo per farlo.»

\section{Il prossimo "Incontro con...»}

Alla fine di ogni mese il Bollettino dei medici svizzeri presenta una personalità che si impegna nel settore sanitario. In agosto Daniel Lüthi vi proporrà un incontro con Pia Coppex, infermiera diplomata e nuova presidentessa della Società Svizzera di Etica Biomedica. 